\title{
Remote Sensing Big Data: Theory, Methods and Applications
}

\author{
Peng Liu ${ }^{1}$, Liping $\mathrm{Di}^{2}$, Qian $\mathrm{Du}^{3}$ and Lizhe Wang ${ }^{1,4, *(1)}$ \\ 1 Institute of Remote Sensing and Digital Earth, Chinese Academy of Science, Beijing 100094, China; \\ liupeng@radi.ac.cn \\ 2 Department of Geography and Geoinformation Science, Center for Spatial Information Science and Systems, \\ George Mason University, Fairfax, VA 22030, USA; ldi@gmu.edu \\ 3 Department of Electrical and Computer Engineering, Mississippi State University, Mississippi State, \\ MS 39759, USA; du@ece.msstate.edu \\ 4 School of Computer Science, China University of Geosciences, Wuhan 430074, China \\ * Correspondence: Lizhe.Wang@gmail.com
}

Received: 2 May 2018; Accepted: 3 May 2018; Published: 4 May 2018

check for updates

\section{Introduction}

Nowadays, our ability to acquire remote sensing data has been improved to an unprecedented level. We have entered an era of big data. Remote sensing data are clearly showing the characteristics of Big Data. Remote sensing big data are attracting more and more attention from government projects, commercial applications to academic fields.

On March 2012, the United States government proposed the "Big Data" Initiative. It could be the first government project on big data that focuses on improving our ability to extract knowledge from large and complex collections of digital data. For remote sensing big data, one of the most important US government projects is the Earth Observing System Data and Information System (EOSDIS). It provides end-to-end capabilities for managing NASA's Earth science data from various sources. In Europe, "Big Data from Space" conference was organized by the European Space Agency in 2017. It is to stimulate interactions and bring together researchers, engineers, users, infrastructure and service providers, interested in exploiting Big Data from Space. Group on Earth Observations (GEO), the largest intergovernmental multilateral cooperation organization is also promoting the development of big data-in October 2017, the National GEOSS Data Sharing Platform of China Deliverables in the GEO Week 2017.

In the field of commercial applications, Google Earth could be one of the examples of success of remote sensing big data. Many remote sensing applications such as target detection, land-cover, smart city, etc. can be developed easily based on Google Earth. With the DigitalGlobe's Geospatial Big Data platform (GBDX) ecosystem, the DigitalGlobal company (Longmont, CO, USA) is creating building footprints quickly by leveraging machine learning in combination with DigitalGlobe's cloud-based 100 petabyte imagery library. Other large companies such as Microsoft (Redmond, WA, USA) and Baidu (Beijing, China) are all developing their electronic maps that are supported with the remote sensing big data and street views' big data. The commercial applications on big data are changing the life of the people.

In academic fields, "remote sensing big data" is also one of the most popular topics. A lot of top journals have launched their special issues about remote sensing big data. IEEE JSTARS launched the special issue on Big Data in Remote Sensing in 2015. Journal of Applied Remote Sensing launched the special issue on Management and Analytics of Remotely Sensed Big Data in 2015. IEEE Geoscience and Remote Sensing Magazine launched the special issue on Big Data from Space in 2016. GeoInformatica of Springer launched the special issue on Big Spatial and Spatiotemporal Data Management and 
Analytics in 2016. Environmental Remote Sensing launched the special issue on Big Remotely Sensed Data: Tools, Applications and Experiences in 2017. Remote Sensing MDPI is calling for papers on special issues on Advanced Machine Learning and Big Data Analytics in Remote Sensing for Natural Hazards Management, SAR in the Big Data Era and Analysis of Big Data in Remote Sensing in 2018. The International Journal of Digital Earth is calling for papers for the special issue on Social Sensing and Big Data Computing for Disaster Management in 2018.

No matter whether it is government projects, commercial applications or academic research, when characterizing big data, it is popular to refer to the 3Vs, i.e., remarkable growth in Volume, Velocity and Variety of data [1]. For remote sensing big data, they could be more concretely extended to characteristics of multi-source, multi-scale, high-dimensional, dynamic-state, isomer, and nonlinear characteristics. It is important for us to consider these more concrete and particular characteristics of remote sensing big data when using remote sensing to extract information and understand geo-processes. It is both an opportunity and a challenge for remote sensing communities. With these issues in mind, it is to present the current state-of-the-art theoretical, methodological, and applicational research on remote sensing big data.

\section{Overview of Contributions}

In this special issue on big data in Remote Sensing, we make an attempt to introduce the latest theory, methods and applications to manage, exploit and analyze remote sensing big data. It contains eight papers that show us some of the latest research advances in big data in remote sensing. To deeply understand big data, we can never neglect the importance of the fundamental theory of data science. Dimension reduction and correlation analysis are two key theory problems of big data. Therefore, we include two papers showing innovations to address the theoretical problem of dimension reduction and multiscale correlatons. Since there are high-dimensional characteristics in hyperspectral images, a graph-based discriminant analysis is proposed in [2] to promote the performance of hyperspectral image dimension reduction. By considering spectral similarities, it avoided insufficient correlations with intrinsic spectral variation of a material by Euclidean distance and formed a appropriate graph representation. To tackle the difficulties of scene classification for High-Resolution Satellite (HRS) images, in [3], the authors use a convolutional neural network to learn and characterize the dense convolutional descriptors at different scales. Then, an adaptive vector quantization termed multiscale correlatons is applied to encode the spatial arrangement of visual words at different scales, and it achieves promising classification results. The improvements for the methods of target detection and object detection based on remote sensing data are also included in this special issue. In [4], to narrow the search region, entropy information was introduced into the combined saliency model to prescreen ship candidates from across the entire maritime domain. Heavy clouds, islands, and ship wakes are used as typical negative samples and constructed the features by gradients to improve the discrimination ability of the detection model. Despite the progress made in natural image object detection fields, the complexity of remote sensing big data makes it difficult to directly deal with the HSR remote sensing imagery object detection task. In [5], a highly efficient and robust integrated geospatial object detection framework based on a faster region-based convolutional neural network is implemented and it improved the performance of geospatial object detection. The problem of finite labeled samples seriously limit the method of deep learning in remote sensing big data. In [6], an improved pre-trained AlexNet architecture (AlexNet-SPP-SS) was proposed. The AlexNet-SPP-SS incorporated the scale pooling - spatial pyramid pooling and side supervision to improve the utilizability of multi-source information fusion in HSR scene classification.

The theory and methods can not change the wold if they are not connected with concrete applications. Thus, this special issue included three papers of applications based on remote sensing big data: flood response decision [7], land-cover change detection [8] and coastal land use [9]. In [7], to optimize the efficiency of the geospatial service in the flood response decision-making system, a parallel agent-as-a-service (P-AaaS) method is proposed and implemented in the cloud computing 
platform. The P-AaaS method includes both parallel architecture and a mechanism for adjusting the computational resources and the execution algorithm. DEM data, transportation and road data, flood depth, topographic map, Landsat TM data, etc. are all used in this application. As the multi-scale and dynamic-state characteristics are considered, in [8], sub-pixel land-cover change detection with the use of different resolution images is addressed. A novel approach based on a back propagation neural network with different resolution images is proposed to overcome problems of mixed pixels in change detection. In this application, both MODIS and Landsat data are used. In [9], the study presents a model named ARCLUSSM (Association Rules-based Coastal Land use Spatial Sequence Model) to mine the sequential pattern of land use with interesting associations in the sea-land direction of the coastal zone. It is a good application of remote sensing big data, which focuses on land use in the sea-land direction and the sequential relationship between land-use types.

\section{Conclusions}

This special issue provides an overview of the research on remote sensing big data from theory, methods to applications. The included eight papers effectively explained the characteristics of multi-source, multi-scale, high-dimensional, dynamic-state, isomer, and nonlinear in remote sensing big data. The research in [5,7] is related to the multi-source and isomer characteristics. The high-dimensional characteristic was addressed in [2] through dimension reduction. The multi-scale and dynamic-state characteristics are utilized in change detection in [8]. The nonlinear characteristic was reflected in the multi-scale correlatons [3] and land use sequential pattern [9]. Thanks to the high quality of the eight selected papers, we believe that the special issue provides the reader with a clear perspective of the current state of remote sensing big data, which is now fully entering the Big Data era. A special thank you goes to all the reviewers. With their constructive comments and professional support, we make this special issue possible.

Acknowledgments: This study is supported by the National Natural Science Foundation of China (No. 41571413 and No. 41471368). We would like to thank the staffs in the editorial office and all the authors who contribute to the special issue.

Conflicts of Interest: The authors declare no conflict of interest.

\section{References}

1. Laney, D. 3D Data Management: Controlling Data Volume, Velocity and Variety. Available online: https:/ / blogs.gartner.com/doug-laney / files/2012/01/ad949-3D-Data-Management-Controlling-DataVolume-Velocity-and-Variety.pdf (accessed on 6 Feburary 2001).

2. Feng, F.; Li, W.; Du, Q.; Zhang, B. Dimensionality Reduction of Hyperspectral Image with Graph-Based Discriminant Analysis Considering Spectral Similarity. Remote Sens. 2017, 9, 323. [CrossRef]

3. Qi, K.; Yang, C.; Guan, Q.; Wu, H.; Gong, J. A Multiscale Deeply Described Correlatons-Based Model for Land-Use Scene Classification. Remote Sens. 2017, 9, 917. [CrossRef]

4. Xu, F.; Liu, J.; Sun, M.; Zeng, D.; Wang, X. A Hierarchical Maritime Target Detection Method for Optical Remote Sensing Imagery. Remote Sens. 2017, 9, 280. [CrossRef]

5. Han, X.; Zhong, Y.; Zhang, L. An Efficient and Robust Integrated Geospatial Object Detection Framework for High Spatial Resolution Remote Sensing Imagery. Remote Sens. 2017, 9, 666. [CrossRef]

6. Han, X.; Zhong, Y.; Cao, L.; Zhang, L. Pre-Trained AlexNet Architecture with Pyramid Pooling and Supervision for High Spatial Resolution Remote Sensing Image Scene Classification. Remote Sens. 2017, 9, 848. [CrossRef]

7. Tan, X.; Guo, S.; Di, L.; Deng, M.; Huang, F.; Ye, X.; Sun, Z.; Gong, W.; Sha, Z.; Pan, S. Parallel Agent-as-a-Service (P-AaaS) Based Geospatial Service in the Cloud. Remote Sens. 2017, 9, 382. [CrossRef] 
8. Wu, K.; Du, Q.; Wang, Y.; Yang, Y. Supervised Sub-Pixel Mapping for Change Detection from Remotely Sensed Images with Different Resolutions. Remote Sens. 2017, 9, 284. [CrossRef]

9. Ding, Z.; Liao, X.; Su, F.; Fu, D. Mining Coastal Land Use Sequential Pattern and Its Land Use Associations Based on Association Rule Mining. Remote Sens. 2017, 9, 116. [CrossRef]

(C) 2018 by the authors. Licensee MDPI, Basel, Switzerland. This article is an open access article distributed under the terms and conditions of the Creative Commons Attribution (CC BY) license (http:// creativecommons.org/licenses/by/4.0/). 